\title{
The Heart Remembers: Anterior T Wave Inversions in a Patient with Intermittent Left Bundle Branch Block
}

\author{
Janaki Rami Reddy Manne ${ }^{1,2^{*}}$ \\ ${ }^{1}$ Department of Hospital Medicine, Marshfield Clinic, USA \\ ${ }^{2}$ University of Wisconsin School of Medicine and Public Health, USA
}

*Corresponding author: Janaki R Manne, MD, Department of Hospital Medicine, Marshfield Clinic, $1000 \mathrm{~N}$ Oak Ave, Marshfield, WI 54449, USA, Tel: 715-387-5127, E-mail: jrrmanne@gmail.com

\begin{abstract}
Even though the new onset of $T$ wave inversions on the electrocardiogram is always an alarming finding, but they are not always pathognomonic of myocardial ischemia. Many cardiac and non-cardiac conditions have been described in association with $\mathrm{T}$ wave inversions on the electrocardiogram. Cardiac memory is a phenomenon characterized by the development of altered T wave polarity on the electrocardiogram when the intrinsic rhythm resumes after a period of abnormal myocardial activation. Misdiagnosis of this condition often leads to unwarranted diagnostic workup and therapeutic interventions. Herein we report a case of 81-year-old female patient with intermittent left bundle branch block presenting with new onset $\mathrm{T}$ wave inversions in anterior leads.
\end{abstract}

\section{Keywords}

Cardiac memory, T wave inversions, Left bundle branch block

\section{Introduction}

Cardiac memory is a specialized form of electrophysiological remodeling characterized by the development of altered $T$ wave polarity on the electrocardiogram when the intrinsic rhythm resumes after a period of abnormal myocardial activation. The resumed sinus or the intrinsic rhythm T wave tracks the QRS vector of the preceding abnormal impulse. This results in the appearance of negative $T$ waves on the electrocardiogram in leads that had negative wide QRS complexes. Misinterpretation of this alarming $T$ wave inversion electrocardiographic pattern by the clinicians will often result in unnecessary work up and treatment. Herein we report a case of cardiac memory in a patient with intermittent left bundle branch block presenting with T wave inversions in anterior leads.

\section{Case Presentation}

An 81-year-old female with dyslipidemia and hypertension presented with a two day history of aching left arm and left shoulder pain. She denied any other symptoms. Upon admission she was registering a blood pressure of $140 / 66 \mathrm{mmHg}$, heart rate of 90 beats $/ \mathrm{min}$, respiratory rate of $24 / \mathrm{min}$ and oxygen saturation of $95 \%$ on room air. Physical examination was unremarkable except for tenderness and limited range of motion at the left shoulder. Admission ECG obtained (Figure 1) demonstrates sinus rhythm with a rate of 90 beats/minute and T wave inversions in the anterior leads V1-V3. An ECG (Figure 2) obtained 2 months ago showed sinus rhythm with a rate of 100 beats/min and left bundle branch block.

Serial troponin I levels (ng/L) measured were less than $16 \mathrm{ng} / \mathrm{L}$ (lab reference $0-45 \mathrm{ng} / \mathrm{L}$ ). All laboratory data including the electrolytes and D-dimer were within normal limits. She had a cardiac catheterization two months ago for chest pain and shortness of breath which showed no coronary artery disease. A transthoracic echocardiogram revealed normal ejection fraction with no wall motion abnormalities. An X-ray of left shoulder joint showed degenerative osteoarthritis and with intraarticular steroid injection her pain significantly improved. A repeat ECG (Figure 3) obtained on the next day showed complete resolution of the anterior T wave inversions and resumption of left bundle branch block.

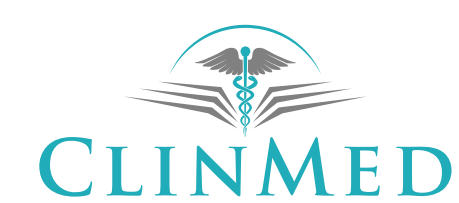

INTERNATIONAL LIBRARY
Citation: Manne JRR (2017) The Heart Remembers: Anterior T Wave Inversions in a Patient with Intermittent Left Bundle Branch Block. Int J Clin Cardiol 4:105. doi.org/10.23937/2378-2951/1410105 Received: September 08, 2017; Accepted: October 09, 2017; Published: October 11, 2017

Copyright: (C) 2017 Manne JRR. This is an open-access article distributed under the terms of the Creative Commons Attribution License, which permits unrestricted use, distribution, and reproduction in any medium, provided the original author and source are credited. 


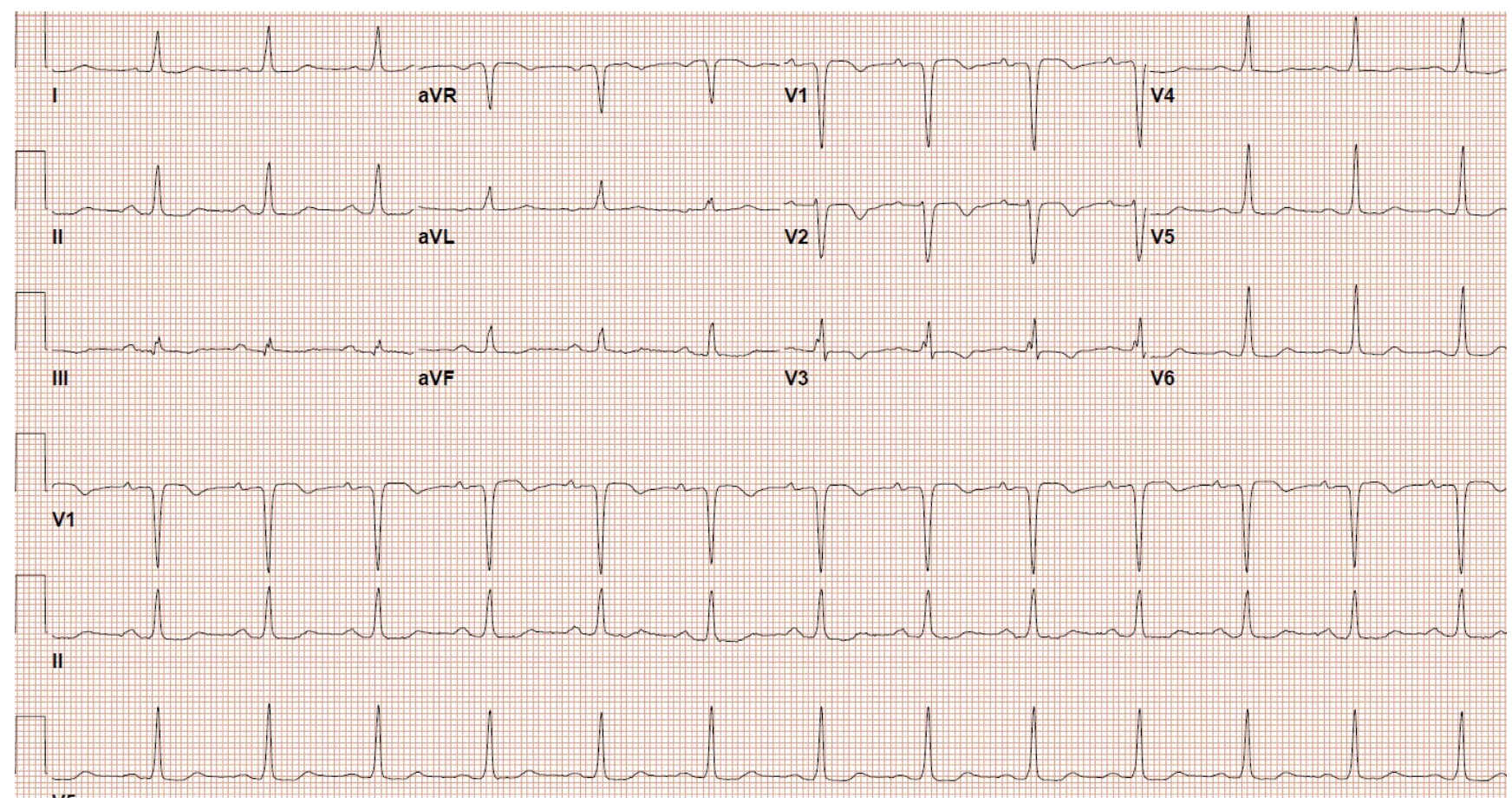

Figure 1: Admission ECG showing T wave inversions in leads V1-V3.

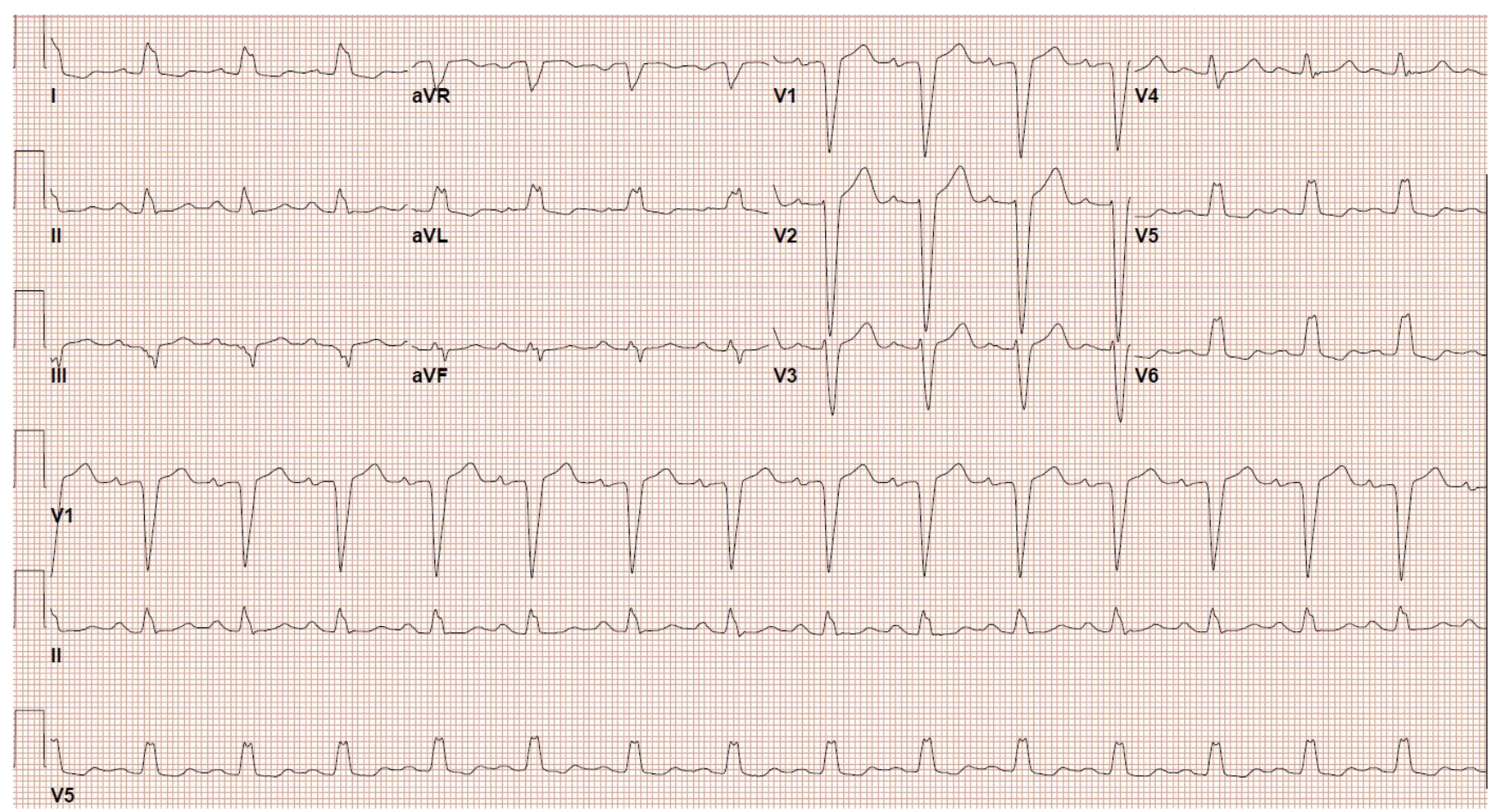

Figure 2: ECG two months prior to this admission showing left bundle branch block and negative QRS complexes in leads that had new $\mathrm{T}$ wave inversions (V1-V3).

In our patient the $\mathrm{T}$ wave inversions were consistent with the diagnosis of cardiac memory due to intermittent left bundle branch block.

\section{Discussion}

Cardiac memory is characterized by diffuse $T$ wave inversions on the electrocardiogram when sinus or any intrinsic rhythm with normal ventricular activation resumes after a period of altered myocardial activation. The resumed sinus or the intrinsic rhythm $T$ wave tracks the QRS vector of the preceding abnormal impulse [1].
In this case, the QRS complexes were negative in leads V1 through V3 with left bundle branch block. The patient's new $T$ wave inversions after resumption of intrinsic rhythm without left bundle branch block mirror these wide QRS complexes by maintaining identical polarity. The phenomenon of Cardiac memory has been observed since 1940s, however the reversible T-wave inversions after a period of ventricular pacing was initially described by Chatterjee, et al. [2] and was named as "cardiac memory" by Rosenbaum, et al. [3]. Cardiac memory is seen in association with intermittent left 


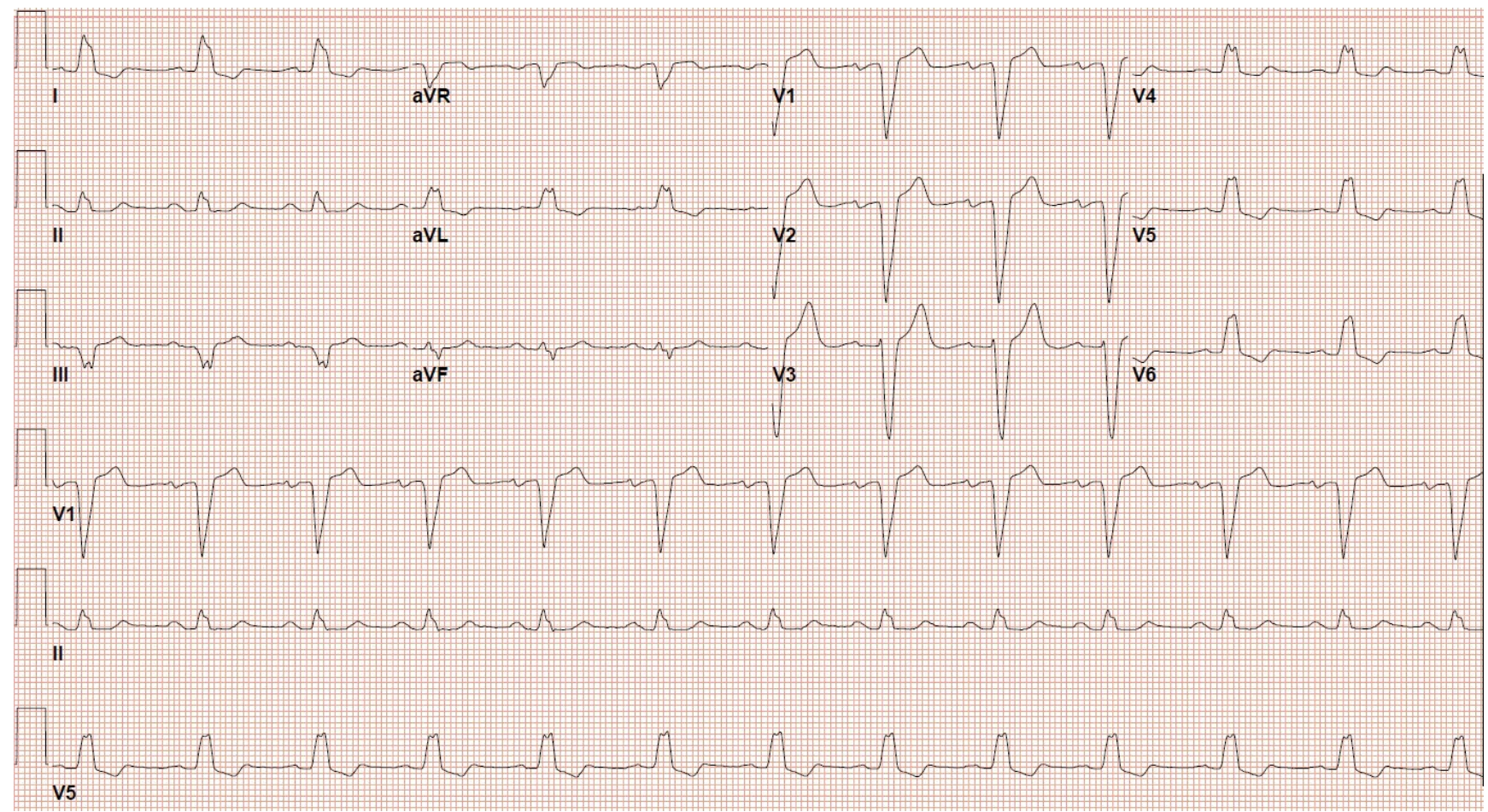

Figure 3: ECG obtained on day 2 showing resumption of left bundle branch block and resolution $T$ wave inversions in leads V1-V3.

bundle branch block, ventricular extrasystoles [4,5], ventricular tachycardia, post-cardio version of ventricular tachycardia, and ventricular pre-excitation/ablation. However the most common cause of $T$ wave memory is ventricular pacing $[2,6]$. The longer the duration and frequency of ventricular pacing the longer the memory T waves persist; this phenomenon is also referred to as "accumulation" [7]. T-wave changes lasting minutes to hours be described as short-term memory, in contrast, T-wave changes lasting hours to days referred to as long-term memory [7]. Changes in the transient outward potassium current (Ito current) is the most studied and reported mechanism. The morphology of the QRS/T ratio determines the magnitude of the $T$-wave vector change seen with cardiac memory; and usually an inverse correlation is seen between the magnitude of the cardiac memory T-wave vector and the QRS/T-vector magnitude ratio [8].

Cardiac memory is a diagnosis of exclusion since many other clinicopathological conditions can present with deep T wave inversions [9]. The common causes of T wave inversions include right/left ventricular overload, Wellen's T waves (proximal left anterior descending coronary artery occlusion), hypertrophic cardiomyopathy, takotsubo cardiomyopathy, acute cerebrovascular events, myopericarditis and pulmonary embolism. Careful history and clinical context will aid in correct diagnosis of T wave inversions avoiding unnecessary work-up and diagnostic interventions. Our patient didn't have signs or symptoms suggestive of any acute intracranial events. Absence of ventricular hypertrophy and wall motion abnormalities on the echocardiogram ruled out hypertrophic and stress induced cardiomyopathy in our patient. Normal appearing pericardium without ventricular dilation and absence of evolutionary EKG changes helped us to exclude pericarditis and myocarditis from the differential diagnosis. Pulmonary embolism is unlikely to be present in our patient considering a normal D-dimer and absence of right ventricular strain on the echocardiogram.

The morphology of T wave can be helpful to differentiate T wave inversions due to myocardial ischemia from cardiac memory after right ventricular pacing. According to a study by Shvilkin, et al. [10] the combination of (1) Positive T wave in lead aVL, (2) Positive or isoelectric T wave in lead I, and (3) Maximal precordial T wave inversion in precordial leads $>$ T wave inversion in lead IIII was $92 \%$ sensitive and $100 \%$ specific for cardiac memory induced by right ventricular pacing, discriminating it from ischemic precordial T wave inversion. However caution should be exercised as these criteria are only applicable in case of cardiac memory from right ventricular pacing but not for the other causes of cardiac memory.

Cardiac memory is a benign condition and T wave inversions due to cardiac memory per se do not require any specific treatment. The $T$ wave inversions in our patient can be explained by Cardiac memory secondary to intermittent left bundle branch block, so no further treatment was pursued.

\section{Conclusion}

Cardiac memory is a well recognized phenomenon but infrequently considered in the differential diagnosis of $T$ wave inversions. Clinicians should be aware of this phe- 
nomenon when interpreting ECG'S with T wave inversions especially in the setting of altered myocardial activation. Cardiac memory is a diagnosis of exclusion and it in no way obviates the need to look for other causes of $\mathrm{T}$ wave inversions. Careful history and clinical context will aid in appropriate workup and correct diagnosis of T wave inversions.

\section{Conflict of Interest}

None declared.

\section{Funding Support}

None.

\section{References}

1. Patberg KW, Shvilkin A, Plotnikov AN, Chandra P, Josephson ME, et al. (2005) Cardiac memory: Mechanisms and clinical implications. Heart Rhythm 2: 1376-1382.

2. Chatterjee K, Harris AM, Davies JG, Leatham A (1969) T-wave changes after artificial pacing. Lancet 1: 759-760.

3. Rosenbaum MB, Blanco HH, Elizari MV, Lazzari JO, Davidenko JM (1982) Electrotonic modulation of the T wave and cardiac memory. Am J Cardiol 50: 213-222.
4. White $P(1950)$ Alteration of the pulse: A common clinical condition. Am J Med Sci 150: 82-96.

5. Harold D Levine, Bernard Lown, Richird B Streeper (1952) The clinical significance of post extra systolic T-wave changes. Circulation 6: 538-548.

6. Erdogan O (2006) Spontaneous T wave inversion after ventricular pacing: What is the probable mechanism? Postgrad Med J 82: e20-e21.

7. Jeyaraj D, Ashwath M, Rosenbaum DS (2010) Pathophysiology and clinical implications of cardiac memory. Pacing Clin Electrophysiol 33: 346-352.

8. Shvilkin A, Bojovic B, Vajdic B, Gussak I, Zimetbaum P, et al. (2009) Vectorcardiographic determinants of cardiac memory during normal ventricular activation and continuous ventricular pacing. Heart Rhythm 6: 943-948.

9. Walder LA, Spodick DH (1993) Global T wave inversion: long-term follow-up. J Am Coll Cardiol 21: 1652-1656.

10. Shvilkin A, Ho KK, Rosen MR, Josephson ME (2005) T-vector direction differentiates post pacing from ischemic T-wave inversion in precordial leads. Circulation 111: 969-974. 